\title{
Management of Electronic Journals using Fuzzy AHP Methodology
}

\author{
N. M. Stefano, N. Casarotto Filho and R. Barichello
}

\begin{abstract}
This work aims to evaluate the management of electronic scientific journals using the IC approach. A hybrid mathematical methodology with the integration of Fuzzy Analytic Hierarchy process (FAHP) was used. The FAHP method was applied to calculate the relative weights of the criteria/sub-criteria selected that affect their management. The results indicated that the Human Capital was appointed as one of the aspects that most $(60 \%)$ influences the management of a periodical. Regarding criteria, the most influential ones are Article normalization; Article quality; Knowledge of those involved; Recognition for the work of referees and contributors; Teamwork Accuracy of published information and Visibility. So, this work is expected to contribute to improvement in the management of electronic scientific journals and to provide competitive advantages.
\end{abstract}

Keywords - FAHP, intellectual capital, electronic journals.

\section{INTRODUÇÃO}

$\mathrm{P}$ OR meio de mobilidade global das informações a força de trabalho, o conhecimento e a proficiência podem ser levadas, instantaneamente, a todo o mundo. Agora, estamos numa sociedade da informação, a economia do conhecimento (dos intangíveis). A geração do conhecimento está fortemente relacionada com as ações desenvolvidas individualmente. Desta forma é importante ressaltar o papel desempenhado pelas organizações que, de uma forma geral, devem estar ligada com as necessidades dos indivíduos. Como resultado, será possível a construção de uma base de conhecimento que não esteja individualizado em cada um de seus colaboradores e sim, disseminado de forma coletiva, em dos níveis hierárquicos existentes.

A premissa é que o conhecimento e a informação estão cada vez mais presentes em nossas vidas, pois aparecem inseridos em todas as nossas ações do dia-a-dia. Isso sucinta o conhecimento à forma útil, na perspectiva do capital. Pois, conhecimento não é soma, é agregação, interação, acumulação.

Como exemplo de interação, disseminação e troca do conhecimento (informação científica) pode-se mencionar o exemplo de periódicos científicos [1], sejam impressos ou eletrônicos, por meio de publicações científicas. As publicações científicas são conteúdos especializados resultantes de informações e procedimentos técnicos, talento, restrições, experiência acumulada, atitudes, normas e valores. Configura-se em produto da ação organizacional, resultando da conjunção de variáveis que integram a atividade humana associada e organizada.

N. M. Stefano, Universidade Federal de Santa Catarina (UFSC), Brazil, stefano.nara@gmail.com

N. Casarotto Filho, Universidade Federal de Santa Catarina (UFSC), Brazil, ncasarottofilho@gmail.com

R. Barichello, Universidade Federal de Santa Catarina (UFSC), Brazil, rodrigobarichello@gmail.com
Em um periódico cientifico, não basta conhecer o fluxo para que se alcance o resultado esperado. Pois, é necessário saber operacionalizá-lo para alcançar a eficiência. Por se tratar de um processo que envolve vários atores - editores, revisores, autores - e como se está lidando com o mundo das ideias, é natural esperar-se que, apesar de sistemático, esse processo contenha uma alta dose de subjetividade [3].

Trzesniak (2006) [3] sugere que o ideal é que os periódicos possuam um manual do processo editorial, contendo a descrição minuciosa de cada procedimento da rotina operacional. Isso, além de facilitar a gestão, garante a uniformidade e, principalmente, a continuidade do processo ao longo do tempo, particularmente quando há uma substituição na equipe (até mesmo do próprio editor). O processo de gestão de um periódico científico eletrônico envolve muitas atividades, algumas, tais como: garantir a qualidade como um todo, cumprimento das normas nacionais e internacionais para sua apresentação, análise de conteúdo, busca de recursos e parcerias, decisões editorias, entre tantas outras.

Neste contexto, esta pesquisa trata de avaliar a gestão de periódicos científicos eletrônicos utilizando a visão de $\mathrm{CI}$. O interesse neste tema reside no fato de que ainda não há um consenso de um modelo definitivo da avaliação de intangíveis em periódicos científicos eletrônicos. As relações com os referees, autores, leitores, fornecedores, parceiros, entre outros são valiosos ativos para periódicos científicos que são incorporados ao capital relacional. A importância do capital relacional como um fator de sustentabilidade talvez seja mais importante em um periódico, seja eletrônico ou impresso do que em outro tipo de serviço do gênero.

As necessidades de informação da comunidade acadêmico/científica, as exigências institucionais estão cada vez mais obrigando os periódicos científicos a participarem de bases dados, diretórios, bibliotecas, repositórios, etc. O serviço que um periódico científico proporciona depende fortemente dos ativos intangíveis (CI); haja vista que o entendimento de sua natureza dinâmica e a forma de geri-los possibilitará o seu crescimento e aumento da competitividade.

A avaliação da gestão dos periódicos científicos eletrônicos por meio de uma de CI permitirá a identificação dos componentes-chave que contribuem para $o$ desenvolvimento e crescimento destes. Quanto ao uso das ferramentas para analisar os dados e estruturar a metodologia, abordagem híbrida FMCDA. Ou seja, Fuzzy AHP (FAHP) justifica-se pelo fato de que: este método é o mais difundindo na literatura e a lógica fuzzy compensa a sua imprecisão e incerteza existentes que é originada no julgamento do decisor(s).

Uma vez que a comparação par-a-par no AHP convencional é insuficiente e imprecisa para capturar o grau de importância do decisor(es) sobre a avaliação de alternativas. Por isso, a lógica fuzzy é introduzido na comparação par-a-par do AHP. A aplicação da teoria dos 
conjuntos fuzzy permite incorporar na imprecisão termos linguísticos que facilitam o processo de decisão.

\section{AVALIAÇÃO DE PERIÓDICOS CIENTÍFICOS}

A avaliação de periódicos científicos é tema de pesquisa desde pelo menos 1960 [4], envolvendo parâmetros para dimensionar a qualidade das informações registradas. $\mathrm{O}$ estudo realizado por Krzyzanowski e Ferreira (1998), que considera os periódicos científicos sob os aspectos de forma e mérito (desempenho do conteúdo), hoje serve como referência para a CAPES (Coordenação de Aperfeiçoamento de Pessoal de Nível Superior), do Ministério da Educação (MEC) para a classificação das publicações.

As primeiras tentativas de avaliação de periódicos, considerando o exame de indicadores de qualidade, datam do início da década 1960, a partir de um modelo desenvolvido pela UNESCO (United Nations Education Science and Culture Organization). No Brasil, algumas tentativas para desenvolver um sistema eficiente de avaliação de periódicos têm sido realizadas. A avaliação de periódicos científicos e técnicas surgem da necessidade de se definirem parâmetros mensuráveis, que possam refletir a qualidade da informação registrada. Essas avaliações, no Brasil, são realizadas pelas comissões de área da CAPES, instituição responsável pela avaliação dos cursos de pós-graduação e da produção científica das universidades [5], [6].

Os critérios Qualis de avaliação é o conjunto de procedimentos utilizados pela CAPES para estratificação da qualidade da produção intelectual dos programas de pósgraduação. Tal processo foi concebido para atender as necessidades específicas do sistema de avaliação e é baseado nas informações fornecidas por meio da coleta de dados. Como resultado, disponibiliza-se uma lista com a classificação dos veículos utilizados pelos programas de pós-graduação para a divulgação da sua produção. A estratificação da qualidade dessa produção é realizada de forma indireta. O Qualis afere a qualidade dos artigos e de outros tipos de produção, a partir da análise da qualidade dos veículos de divulgação, ou seja, periódicos científicos [7]. A classificação de periódicos é realizada pelas áreas de avaliação e passa por processo anual de atualização. Esses veículos são enquadrados em estratos indicativos da qualidade - A1 (o mais elevado); A2; B1; B2; B3; B4; B5; C (com peso zero) - dentro dos âmbitos, nacional e internacional.

É importante observar que um mesmo periódico, ao ser classificado em duas ou mais áreas distintas, pode receber diferentes avaliações. Segundo a Capes (2012) [7], isto não constitui inconsistência, mas expressa o valor atribuído, em cada área, à pertinência do conteúdo veiculado. Por isso, não se pretende com esta classificação que é específica para o processo de avaliação de cada área, definir qualidade de periódicos de forma absoluta.

Nos periódicos estrangeiros, utiliza-se o Fator de Impacto (FI) da base de dados Journal Citation Reports (JCR) do Institute for Scientific Information (ISI) para os níveis A e B, sendo nível $\mathrm{A}$ os periódicos com FI igual ou superior a $0,5 \mathrm{e}$ nível $\mathrm{B}$ os periódicos com FI inferior a 0,5. No caso de periódicos editados no exterior que não façam parte do JCR, a Comissão de Área analisará outras informações disponíveis, por exemplo, no sítio do periódico, para definir a respectiva classificação. Os FIs são calculados dividindo-se o número de citações feitas no ano corrente a artigos publicados nos dois anos anteriores pelo número total de artigos publicados nos dois anos anteriores. O FI é, assim, uma medida da frequência com que um determinado de um periódico é citado em um ano específico.

Mas, além de proporcionar as informações de citação, o JCR indica, também, a velocidade com que estas citações (de certo periódico) aparecem na literatura, por meio do cálculo do número médio de vezes que um artigo é citado durante o ano que foi publicado [8]. O sistema de citação foi inventado principalmente como uma maneira de entender como as descobertas científicas e inovações são comunicadas e como funcionam [9]. Não foi inicialmente visto como uma ferramenta para avaliar os cientistas de forma individual, universidades ou sistemas acadêmicos. Assim, o sistema de citação é útil para monitorar como as ideias científicas são propagadas entre os pesquisadores e cientistas (individual) e como comunicam os resultados da sua investigação.

O FI dos periódicos está se tornando cada vez mais um parâmetro importante para avaliar revistas em todo o mundo. Além deste método, há o Web Impact Factors (WIF). Ingwersen (1998) [10] propôs WIF como o equivalente do FI do ISI. O WIF baseia-se no número de ligações feitas em $\mathrm{Web}$ Site, em comparação ao tamanho (geralmente o número de páginas) do Site. Ele, também fornece ferramentas quantitativas para a classificação, avaliação, categorização e comparação de Web Sites, domínios e subdomínios. Embora o FI seja a ferramenta mais utilizada na literatura, outras propostas para avaliar periódicos científicos são encontradas na literatura. Destacam-se os critérios de avaliação apresentados por bases de dados como Scientific Eletronic Librarym Online (Scielo), Scopus, Plataforma ISI/web of Knowledge, Periódicos de Acesso Livre (OASIS. Br). O SciELO e o Qualis Capes estabelecem os seus próprios critérios, políticas e procedimentos para a admissão e a continuação de periódicos científicos em sua coleção.

Portanto, não é objetivo deste trabalho apresentar de forma detalhada cada uma destas metodologias propostas para avaliar periódicos científicos. A lição que se tira disto tudo é que não há conformidade sobre quais são os procedimentos ou ferramentas mais adequadas para se avaliar um periódico científico.

Mas, para a realização de qualquer avaliação, é necessário determinar quais parâmetros (mesmo que resultem em concordâncias ou não) que devem ser levados em consideração e quais tipos de dados serão considerados. As abordagens sobre a avaliação dos periódicos científicos são variadas e os modelos sugeridos adotam diferentes critérios.

\section{FUZZY ANAL YTIC HIERARCHY PROCESS (FAHP)}

Zadeh (1965) [11] introduziu a teoria dos conjuntos fuzzy, para racionalizar a incerteza associada com a imprecisão, de forma análoga ao pensamento humano. Assim, o AHP passou a ter uma extensão fuzzy, sendo desenvolvido para resolver problemas de imprecisão hierárquica.

O AHP convencional é inadequado para capturar e explicitar os requisitos do decisor, isto é, ele não pode processar o conhecimento vago ou impreciso. Para modelar este tipo de incerteza (da preferência humana) os conjuntos 
fuzzy podem ser incorporados na comparação par-a-par do AHP. E, portanto, a abordagem Fuzzy AHP permite uma descrição mais precisa do processo decisório [12]. O AHP [13] baseia-se em uma estruturação empírica baseada em conhecimentos dos especialistas que, por meio da atribuição de pesos e critérios decisórios, resulta no apontamento da solução mais interessante ao problema a ser resolvido. Tratase de uma metodologia de análise multicriterial que segue uma estratégia para a redução da complexidade de um problema segundo uma estrutura hierárquica. Esse método usa da capacidade humana de utilizar a informação e a experiência para estimar magnitudes relativas por meio de comparações par-a-par. É uma abordagem flexível que emprega a lógica aliada à intuição, com o objetivo de obter julgamentos pelo consenso. O método AHP [14], [15], envolve três fases para resolver o problema de decisão:

(1) Decomposição - é a construção hierárquica para representar um problema de decisão, no topo é colocado o objetivo global e nos níveis mais baixos estão os critérios, subcritérios e alternativas.

(2) Julgamentos comparativos - nesta etapa e solicitado aos participantes de um grupo a construção da matriz de comparação onde são comparados os pares de critérios e subcritérios. É apresentada nessa etapa uma escala de valores que variam de 1 (indiferença) a 9 (preferência extrema) para expressar a preferência de cada componente do grupo de discussão.

(3) Síntese de prioridades - é a fase de calcular um peso para cada alternativa fundamentada em preferências derivadas da matriz de comparação.

O modelo AHP identifica: as áreas de maior e menor oportunidade; prioriza as opções; clarifica as diferenças entre as alternativas; ajuda os envolvidos (atores) a entender melhor uma situação; indica a melhor alocação de recursos para atingir o(s) objetivo(s); facilita a geração de novas e melhores opções e favorece a comunicação entre as partes integrantes do processo. Assim o AHP é uma ferramenta interativa, útil para analistas e tomadores de decisão na resolução de problemas complexos relacionados a interesses em vários contextos.

Na realidade o AHP é um método multicriterial para a resolução de problemas complexos de decisão, que utiliza uma formulação matemática racional para tratar com os aspectos qualitativos que rodeiam as pessoas e os objetos envolvidos. Com a incerteza das informações e a imprecisão do sentimento e reconhecimento humano, é difícil fornecer valores numéricos exatos para os critérios e fazer avaliações que transmitam, exatamente, tais sentimentos para os decisores. Dessa fora, a maioria dos parâmetros de seleção não pode ser dada com precisão. A teoria dos conjuntos fuzzy torna o processo de comparação mais flexível e capaz de explicar as preferências dos decisores [12], [16], [17]. Isto permite que alguns decisores possam preferir julgamentos intermediários, em vez de certas decisões.

Assim, diferentes métodos para a fuzzificação do AHP têm sido propostos na literatura. Estes métodos são abordagens sistemáticas para a seleção de alternativas e justificativas para problemas usando os conceitos da teoria dos conjuntos fuzzy. Em 1979, o próprio Thomas Saaty, criador do AHP, explorou interfaces entre hierarquias, objetivos múltiplos e conjuntos fuzzy, estabelecendo sobre o assunto, provavelmente, as bases para a exploração de outros modelos FAHP.

\section{MODELAGEM MATEMÁTICA}

A modelagem matemática, tem a finalidade de determinar o peso dos critérios/subcritérios que mais influenciam na gestão dos periódicos cientifico eletrônico. Neste trabalho, será utilizada a análise de extensão do FAHP para o cálculo dos pesos dos critérios proposta por Chang (1996) [18]. A utilização do método de Chang é devido ao fato de que os passos desta abordagem são semelhantes ao AHP convencional e relativamente mais fácil do que as outras abordagens FAHP. A escala fuzzy triangular de preferência utilizada neste trabalho é dada pela Tabela I.

\begin{tabular}{cccc} 
TABELA I. RELAÇÕES ENTRE AS VARIÁVEIS LINGUÍSTICAS E \\
\multicolumn{4}{c}{ SUAS FUNÇÕES DE PERTINENCIA. } \\
\hline $\begin{array}{c}\text { Escala linguística de } \\
\text { importância }\end{array}$ & $\begin{array}{c}\text { Números } \\
\boldsymbol{f u z z y} \\
\text { para } \\
\text { FAHP }\end{array}$ & $\begin{array}{c}\text { Escala } \\
\text { triangular } \\
\boldsymbol{f u z z y}(\boldsymbol{l}, \boldsymbol{m}, \boldsymbol{u})\end{array}$ & $\begin{array}{c}\text { Escala recíproca } \\
\text { triangular } \boldsymbol{f u z z y}\end{array}$ \\
\hline Justamente Igual & - & $(1,1,1)$ & \\
Igualmente Importante & 1 & $(0.66,1,1.5)$ & $(0.66,1,1.5)$ \\
Fracamente Importante & 2 & $(1,1.5,2)$ & $(0.5,0.66,1)$ \\
Moderadamente Importante & 3 & $(1.5,2,2.5)$ & $(0.4,0.5,0.66)$ \\
Fortemente Importante & 4 & $(2,2.5,3)$ & $(0.33,0.4,0.5)$ \\
Extremamente Importante & 5 & $(2.5,3,3.5)$ & $(0.29,0.33,0.4)$ \\
\hline
\end{tabular}

A seguir é detalhado o método proposto por Chang (1996) [18], utilizado por Kahraman, Cebeci e Ruan (2004) [19], Kutlu e Ekmekçioğlu (2012) [17], Cho e Lee (2013) [20] e Wang et al. (2014) [21] entre tantos outros pesquisadores. Os passos seguidos para a aplicação do FAHP são:

Passo 1: Formar as comparações de pares dos atributos usando os números fuzzy, o qual é composto por valores baixos, médios e superiores, no mesmo nível da estrutura hierárquica.

Passo 2: O valor da medida sintética fuzzy em relação ao $i^{\text {th }}$ objeto é definida pelas Equações 1, 2, 3, e 4:

$$
\begin{gathered}
S_{i}=\sum_{j=1}^{m} M_{g i}^{j} \otimes\left[\sum_{i=1}^{n} \sum_{j=1}^{m} M_{g i}^{j}\right]^{-1} \\
\sum_{j=1}^{n} M_{i j}=\left(\sum_{j=1}^{n} l_{i j}, \sum_{j=1}^{n} m_{i j}, \sum_{j=1}^{n} u_{i j}\right), i=1,2,3, \ldots, n \\
\sum_{i=1}^{m} \sum_{j=1}^{n} M_{g i}^{j}=\left(\sum_{i=1}^{m} \sum_{j=1}^{n} l_{i j}, \sum_{i=1}^{m} \sum_{j=1}^{n} m_{i j}, \sum_{i=1}^{m} \sum_{j=1}^{n} u_{i j}\right) \\
{\left[\sum_{i=1}^{m} \sum_{j=1}^{n} M_{i j}\right]^{-1}=\left(\frac{1}{\sum_{i=1}^{m} \sum_{j=1}^{n} u_{i j}}, \frac{1}{\sum_{i=1}^{m} \sum_{j=1}^{n} m_{i j}}, \frac{1}{\sum_{i=1}^{m} \sum_{j=1}^{n} l_{i j}}\right)}
\end{gathered}
$$

Passo 3: $\mathrm{O}$ grau de possibilidade de $M_{2}=\left(l_{2}, m, u_{2}\right) \geq$ $M_{1}=\left(l_{1}, m_{1}, u_{1}\right)$ é definido (Equação 5) como:

$$
V\left(M_{2} \geq M_{1}\right)=\sup _{y \geq x}\left[\min \left(\mu_{M_{2}}(x), \mu_{M_{2}}(y)\right)\right]
$$

E, pode ser equivalentemente a Equação 6: 
$V\left(M_{2} \geq M_{1}\right)=\operatorname{hgt}\left(M_{1} \cap M_{2}\right)=\mu_{M_{2}}(d)=\left\{\begin{array}{c}1, \text { se } m_{2} \geq m_{1} \\ 0, \text { se } l_{1} \geq l_{2} \\ \frac{l_{1}-u_{2}}{\left(m_{2}-u_{2}\right)-\left(m_{1}-l_{1}\right)}, c c\end{array}\right.$

Onde $d$ é a é a ordenada (Fig. 1) de maior intersecção ponto $D$, entre $\mu_{M_{1}}$ e $\mu_{M_{2}}$.

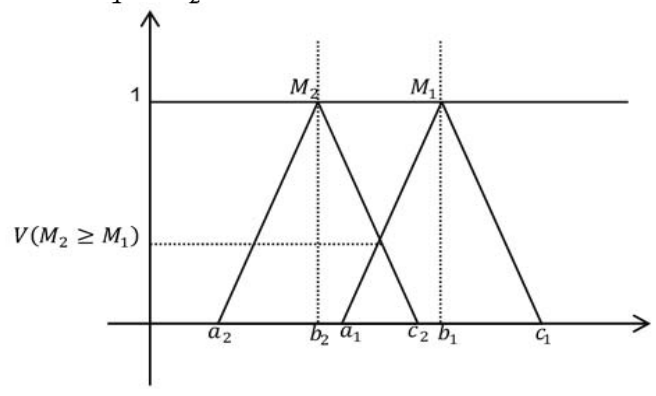

Figura 1. intersecção entre $M_{1}$ e $M_{2}$

Para comparar $M_{1}$ e $M_{2}$ precisa-se os valores de $V\left(M_{1} \geq\right.$ $\left.M_{2}\right)$ e $V\left(M_{2} \geq M_{1}\right)$.

Passo 4: O grau de possibilidade para um número fuzzy convexo ser maior do que $k$ números fuzzy convexos $M_{i}(i=$ $1,2,3, \ldots, k)$ pode ser definido pela Equação 7 .

$V\left(M \geq M_{1}, M_{2}, \ldots, M_{k}\right)=V\left[\left(M \geq M_{1}\right)\right] e\left(M \geq M_{2}\right)$ e $\ldots e\left(M \geq M_{k}\right)=$ $\min V\left(M \geq M_{i}\right), i=1,2,3, \ldots, k \quad(7)$

A Equação 7 assume a forma da Equação 8.

$$
d^{\prime\left(A_{i}\right)}=\min V\left(S_{j} \geq S_{i}\right)
$$

Para $k=1,2, \ldots, n ; k \neq i$. Em seguida, o vetor de peso (Equação 9) é dado por:

$$
W^{\prime}=\left(d^{\prime\left(A_{1}\right)}, d^{\prime\left(A_{2}\right)}, \ldots, d^{\prime\left(A_{n}\right)}\right)^{T}
$$

Onde $A_{i}(i=1,2, \ldots, n)$ tem $n$ elementos.
Passo 5: Por meio da normalização, os vetores de peso são normalizados pela Equação 10. Onde $W$ é um número não fuzzy.

$$
W=\left(d\left(A_{1}\right), d\left(A_{2}\right), \ldots, d\left(A_{n}\right)\right)^{T}
$$

Passo 6: Cálculo os pesos globais [22] para os subcritérios. Os pesos globais de subcritérios são calculados multiplicando-se o peso local dos subcritérios com o peso dos critérios a que pertence. Os pesos globais são denotados por $w_{\text {sub }}^{i}=\left(w_{i 1}, w_{i 2}, \ldots, w_{i n i}\right)$, onde $n_{i}$ é o número de subcritérios com relação ao $i_{n}$ critério.

\section{APLICAÇÃO DO FAHP}

Primeiramente o questionário com os aspectos, critérios e subcritérios, foi simplificado com siglas para melhor trabalhar o método FAHP. A seleção dos periódicos para a pesquisa teve como critério de definição o Qualis Capes (foram utilizados os periódicos com Qualis A1, A2, B1 e B2) que é estipulado pela Capes. Foram selecionados periódicos de diversas áreas (Administração, Engenharia III, Ciências Ambientais, Educação Física, Ensino, Planejamento Urbano e Regional/Demografia). Foram enviados um total de 27 questionários e retornar 14 . Os periódicos eletrônicos que fizeram parte da amostragem foram: Journal of Cleaner Production, Semina. Ciências Agrárias, Transinformação, Iberoamerican Journal of Industrial Engineering (IJIE), Ciências Sociais Unisinos, Custos e @gronegócio Online, Estudos Feministas, Revista em Agronegócios e Meio Ambiente, Revista Colabor@, Revista da Educação física da UEM, Brazilian Journal of Chemical Engineering, Revista Gestão \& Planejamento, Revista Ambiência e Brazilian Journal of Poultry Science).

O modelo AHP é formado pelo(s) objetivo principal, os aspectos, critérios e subcritérios determinados no primeiro passo (Fig. 2). 


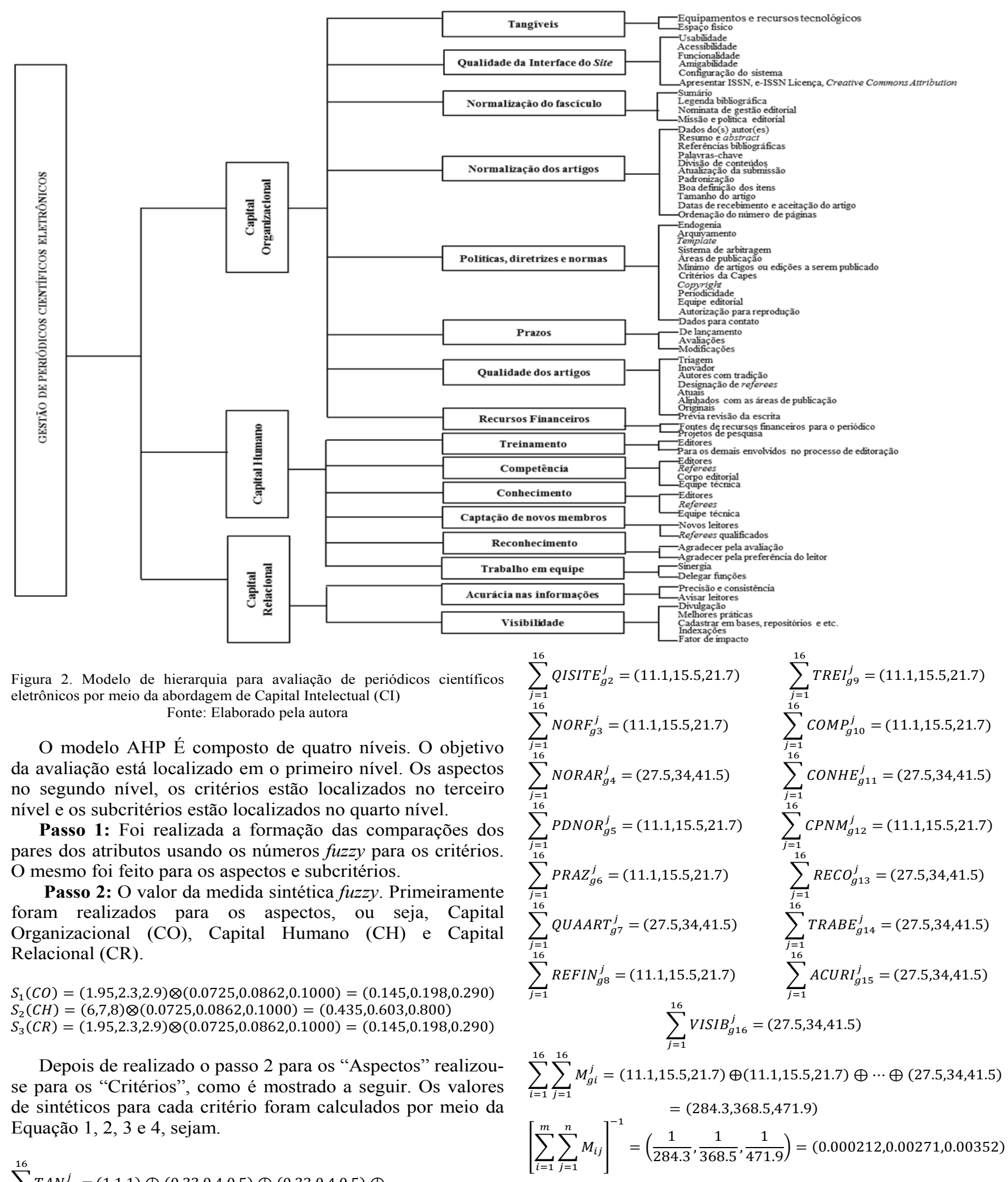

$\sum_{j=1}^{16} T A N_{g 1}^{j}=(1,1,1) \oplus(0.33,0.4,0.5) \oplus(0.33,0.4,0.5) \oplus \ldots$

O mesmo procedimento foi realizado para os subcritérios.

Passo 3: Usando os resultados do passo anterior são obtidos para calcular os vetores peso. De acordo com as 
Equações 5 e 6 o grau de possibilidade de $S_{i}=\left(l_{j}, m_{j}, u_{j}\right) \geq$ $S_{i}=\left(l_{i}, m_{i}, u_{i}\right)$ pode ser calculada por meio da comparação dos valores de $S_{i}$. Calcula-se o grau mínimo de possibilidade $d^{\prime}(i)$ de $V\left(S_{j} \geq S_{i}\right)$ para $i, j=1,2,3, \ldots, k$. Ou seja:

$$
\begin{aligned}
& \left.S_{4}(\text { NORAR })=(0.58,0.0921,0.146)\right) \\
& \left.S_{1}(T A N)=(0.00064,0.0176,0.027)\right\} \\
& V(S 1 \geq S 4)=\frac{0.58-0.027}{(0.0176-0.027)-(0.0921-0.058)}=0.22
\end{aligned}
$$

O mesmo procedimento foi realizado para os subcritérios

Passo 4: Aqui nesta etapa é calculado o grau mínimo de possibilidades $\left(d^{\prime}(i)\right.$ de $\left.\left.V_{j} \geq S_{i}\right)\right)$ para $i, j=1,2,3, \cdots, k$. Como resultado obtém-se o seguinte vetor peso: $(0.22,0.58,0.58,0.58,1,0.58,0.58,1,0.58,0.58,0.58,1,0.58,1,1,1,1)^{T} \quad$ e para os aspectos obteve-se o seguinte: : $(0.80,0.80,1,1)^{T}$. O mesmo procedimento foi feito para os subcritérios.

Passo 5: Normalização dos pesos. Após a normalização do valor desses pesos com relação ao objetivo principal tem-se o seguinte:

$W=(0.018,0.042,0.042,0.092,0.042,0.042,0.092,0.042,0.042,0.042,0.092,0.042,0.092,0.092,0.092,0.092)$

Em seguida, os pesos dos subcritérios são calculados da mesma forma. A Tabela II mostra o peso final de cada critério.

\begin{tabular}{ccc}
\multicolumn{3}{c}{ TABELA II. Pesos do subscritérios. } \\
\hline Aspectos & Peso (W) & W (\%) \\
\hline Capital Organizacional (CO) & 0.20 & 0.20 \\
Capital Humano (CH) & 0.60 & 0.60 \\
Capital Relacional (CR) & 0.20 & 0.20 \\
Total & $\mathbf{1 . 0 0}$ & $\mathbf{1 0 0}$ \\
Critérios & Peso (W) & $\mathbf{W ~ ( \% )}$ \\
Tangíveis (TAN) & 0.018 & 1.8 \\
Qualidade da Interface do Site (QISITE) & 0.042 & 4.2 \\
Normalização do fascículo (NORF) & 0.042 & 4.2 \\
Normalização dos artigos (NORAR) & 0.092 & 9.2 \\
Políticas, diretrizes e normas (PDNOR) & 0.042 & 4.2 \\
Prazos (PRAZ) & 0.042 & 4.2 \\
Qualidade dos artigos (QUAART) & 0.092 & 9.2 \\
Recursos Financeiros (REFIN) & 0.042 & 4.2 \\
Treinamento (TREI) & 0.042 & 4.2 \\
Competências (COMP) & 0.042 & 4.2 \\
Conhecimento (CONHE) & 0.092 & 9.2 \\
Captação de novos membros (CPNM) & 0.042 & 4.2 \\
Reconhecimento (RECO) & 0.092 & 9.2 \\
Trabalho em equipe (TRABE) & 0.092 & 9.2 \\
Acurácia das informações (ACURI) & 0.092 & 9.2 \\
Visibilidade (VISIB) & 0.092 & 9.2 \\
Total & $\mathbf{1 , 0 0}$ & $\mathbf{1 0 0}$ \\
\hline
\end{tabular}

Pelo exposto na Tabela II percebe-se que o aspecto Capital Humano (CH) obteve o maior peso, ou seja, o que mais influência na gestão de um periódico científico eletrônico. De fato, o CH é o "motor" no que tange ao gerenciamento de um periódico científico eletrônico, sem esse fator as atividades não podem ser realizadas e além de ser a principal fonte de vantagem competitiva sustentável.

Passo 6: Cálculo do peso global dos subcritérios. Foi calculado da seguinte forma: por exemplo, o peso global dos subcritério com respeito a "Recursos Financeiros (REFIN)" é $w_{\text {Subc }}^{1}=0,042 \otimes(0,0082,0,0042)=(0,0003437,0,000177)$.

Tabela III mostra os resultados para todos os subcritérios.

\begin{tabular}{|c|c|c|c|c|}
\hline Critérios & Subcritérios & Peso Local & Peso Global & $\begin{array}{c}\text { Peso Global } \\
(\%)\end{array}$ \\
\hline & CONFS & 0.1194 & 0.0050 & 0.50 \\
\hline & APLIC & 0.4032 & 0.0169 & 1.7 \\
\hline \multirow{4}{*}{ NORF } & SUM & 0.25 & 0.0230 & 2.30 \\
\hline & LEGB & 0.25 & 0.0230 & 2.30 \\
\hline & NOMGE & 0.25 & 0.0230 & 2.30 \\
\hline & MPOLE & 0.25 & 0.0230 & 2.30 \\
\hline \multirow[t]{11}{*}{ NORAR } & AFCAU & 0.1575 & 0.0145 & 1.45 \\
\hline & RESAB & 0.1575 & 0.0145 & 1.45 \\
\hline & PREFBI & 0.0524 & 0.0048 & 0.48 \\
\hline & PALCH & 0.1575 & 0.0145 & 1.45 \\
\hline & DIVC & 0.0524 & 0.0048 & 0.48 \\
\hline & ATEDOC & 0.0524 & 0.0048 & 0.48 \\
\hline & PADR & 0.0524 & 0.0048 & 0.48 \\
\hline & BAODF & 0.0524 & 0.0048 & 0.48 \\
\hline & TAMART & 0.0524 & 0.0048 & 0.48 \\
\hline & DRAAR & 0.0524 & 0.0048 & 0.48 \\
\hline & ORDCNP & 0.1575 & 0.0145 & 1.45 \\
\hline \multirow{12}{*}{ PDNOR } & END & 0.18 & 0.0075 & 0.75 \\
\hline & ARQ & 0.068 & 0.0029 & 0.29 \\
\hline & TEMP & 0.068 & 0.0029 & 0.29 \\
\hline & SARBP & 0.18 & 0.0075 & 0.75 \\
\hline & ARPUB & 0.068 & 0.0029 & 0.29 \\
\hline & MINAP & 0.03 & 0.0012 & 0.12 \\
\hline & CRTC & 0.068 & 0.0029 & 0.29 \\
\hline & COPY & 0.068 & 0.0029 & 0.29 \\
\hline & PERIOD & 0.068 & 0.0029 & 0.29 \\
\hline & EQED & 0.068 & 0.0029 & 0.29 \\
\hline & AUTR & 0.068 & 0.0029 & 0.29 \\
\hline & DADC & 0.068 & 0.0029 & 0.29 \\
\hline \multirow{3}{*}{ PRAZ } & LANCED & 0.3333 & 0.0140 & 1.40 \\
\hline & AVAL & 0.3333 & 0.0140 & $\begin{array}{l}1.40 \\
1.40\end{array}$ \\
\hline & MOD & 0.3333 & 0.0140 & 1.40 \\
\hline \multirow{8}{*}{ QUAART } & TRIA & 0.125 & 0.0115 & 1.15 \\
\hline & INOV & 0.125 & 0.0115 & 1.15 \\
\hline & AUTTR & 0.125 & 0.0115 & 1.15 \\
\hline & DEREF & 0.125 & 0.0115 & 1.15 \\
\hline & ATUAIS & 0.125 & 0.0115 & 1.15 \\
\hline & ALCARP & 0.125 & 0.0115 & 1.15 \\
\hline & ORIG & 0.125 & 0.0115 & 1.15 \\
\hline & REVESC & 0.125 & 0.0115 & 1.15 \\
\hline \multirow[t]{2}{*}{ REFIN } & FONTES & 0.71 & 0.0300 & 3.00 \\
\hline & PROJP & 0.29 & 0.0121 & 1.21 \\
\hline \multirow[t]{2}{*}{ TREI } & EDIT1 & 0.50 & 0.2500 & 25.00 \\
\hline & DEENV & 0.50 & 0.2500 & 25.00 \\
\hline \multirow{4}{*}{ COMP } & EDIT2 & 0.25 & 0.1050 & 10.50 \\
\hline & REFER1 & 0.25 & 0.1050 & 10.50 \\
\hline & COPED & 0.25 & 0.1050 & 10.50 \\
\hline & EQTEC1 & 0.25 & 0.1050 & 10.50 \\
\hline \multirow{3}{*}{ CONHE } & EDIT3 & 0.3333 & 0.0307 & 3.07 \\
\hline & REFER2 & 0.3333 & 0.0307 & 3.07 \\
\hline & EQTEC2 & 0.3333 & 0.0307 & 3.07 \\
\hline \multirow[t]{2}{*}{ CPNM } & NOVLA & 0.5 & 0.2500 & 25.00 \\
\hline & REFQ & 0.5 & 0.2500 & 25.00 \\
\hline \multirow[t]{2}{*}{ RECO } & REFER3 & 0.75 & 0.0690 & 6.90 \\
\hline & AGRLE & 0.25 & 0.0230 & 2.30 \\
\hline \multirow[t]{2}{*}{ TRABE } & SINER & 0.75 & 0.0690 & 6.90 \\
\hline & DELF & 0.25 & 0.0230 & 2.30 \\
\hline \multirow[t]{2}{*}{ ACURI } & PRECEC & 0.75 & 0.0690 & 6.90 \\
\hline & AVISL & 0.25 & 0.0230 & 2.30 \\
\hline \multirow{5}{*}{ VISIB } & DIVUL & 0.20 & 0.0184 & 1.84 \\
\hline & MELPR & 0.2 & 0.0184 & 1.84 \\
\hline & CABRE & 0.2 & 0.0184 & $\begin{array}{l}1.07 \\
1.84\end{array}$ \\
\hline & INDEX & 0.2 & 0.0184 & 1.84 \\
\hline & FATIM & 0.2 & 0.0184 & 1.84 \\
\hline
\end{tabular}

TABELA III. Peso global dos subcritérios.

\begin{tabular}{c|c|c|c|c}
\multicolumn{5}{c}{ TABELA III. Peso global dos subcritérios. } \\
\hline \multirow{2}{*}{ Critérios } & Subcritérios & Peso Local & Peso Global & $\begin{array}{c}\text { Peso Global } \\
(\mathbf{\%})\end{array}$ \\
\hline \multirow{3}{*}{ TAN } & EQP RT & 0.71 & 0.0129 & 1.29 \\
\cline { 2 - 5 } & INSTF & 0.29 & 0.0051 & 0.51 \\
\hline \multirow{3}{*}{ QISITE } & USAB & 0.1194 & 0.0050 & 0.50 \\
\cline { 2 - 5 } & ACESS & 0.1194 & 0.0050 & 0.50 \\
\cline { 2 - 5 } & FUNC & 0.1194 & 0.0050 & 0.50 \\
\cline { 2 - 5 } & AMIGC & 0.1194 & 0.0050 & 0.50 \\
\hline
\end{tabular}

Como mostra a Tabela III o subcritérios com maior peso foi APLIC (0.4032) (apresentar International Standard Serial Number - ISSN, e-ISSN Licença, Creative Commons Attribution), esse é um fator muito importante em se tratando da gestão de periódicos científicos eletrônicos, neste caso bem como para os impressos. 


\section{CONCLUSÕES E RECOMENDAÇÕES}

Portanto, espera-se que esse trabalho possa auxiliar os editores a repensar e a conduzir a gestão de seus periódicos. Em se tratando de gestão de periódicos científicos, seja eletrônico ou impresso, o acesso aberto ou restrito, o aspecto mais importante a se destacar é sua sustentabilidade. Seja ela referente aos aspectos econômicos ou as submissões de artigos, pois um periódico científico sem artigos praticamente não existe. A sustentabilidade do periódico científico eletrônico não se refere apenas a aspectos econômicos do processo de edição e publicação online. Juntamente com os custos, está a acessibilidade, a recuperação de informações, navegabilidade e interatividade. Ou seja, características da Tecnologia da Informação e Comunicação (TICs), especificamente a Internet. Pois, o desenvolvimento da ciência influência de forma direta a comunicação científica, de certa forma podendo refletir na circulação do conhecimento originado por esta atividade sendo inerente à sua própria concepção. O processo publicação de um periódico científico eletrônico exige que se garanta a qualidade dos conteúdos publicados tendo como referência os valores das diferentes áreas da ciência, dentro de um sistema de comunicação que está em constante modificação. Para trabalhos futuros recomenda-se a reaplicação e o acompanhamento dessa proposta em um ou mais periódicos científicos eletrônicos. Ainda sugere-se:

- A utilização de outros métodos de avaliação multicritérios para avaliar a gestão tais como: TOPSIS (Technique for Order of Preference by Similarity to Ideal Solution), PROMETHEE (Preference Ranking Organization Method for Enrichment of Evaluations) Methods, ELECTRE family.

- Utilizar uma amostragem maior (acima de 100) e utilizar estatística multivariada (ou equações estruturais) para observar o comportamento desses periódicos quanto a sua gestão.

- Propor uma visão de qualidade de serviços para avaliar a qualidade dos periódicos científicos eletrônicos.

Também seria interessante avaliar e priorizar na visão do comportamento dos 7M's (Management, Manpower, Marketing, Method, Machine, Material, and Money) para melhorar o desempenho desses periódicos.

\section{REFERÊNCIAS}

1] N.M. Stefano, J.V. Souza, F.K. Merlin, L. Ensslin and S.R. Ensslin. Modelo para avaliar a revista Iberoamericana de Engenharia Industrial tendo em vista sua gestão. Revista Gestão Industrial, v. 7, n. 4, p. 54-82, 2011

[2] K.M. Kacmar. Setting up an effective manuscript-review process. In: Y. Baruch, A.M. Konrand, H. Aguinis and W.H. Starbuck (ed). Opening the black box of editorship. New York: Palgrave, 2008.

[3] P. Trzesniak. As dimensões da qualidade dos periódicos científicos e sua presença em um instrumento da área da educação. Revista Brasileira de Educação, v. 11, n. 32, p. 346-361, 2006.

[4] A.C. Gruszynski. A edição de periódicos científicos eletrônicos: desafios para a visibilidade da ciência na Web. In: INTERCOM - Sociedade Brasileira de Estudos Interdisciplinares da Comunicação XXX Congresso Brasileiro de Ciências da Comunicação - Anais..., 2007.

[5] G.R.B. Fachin. Modelo de avaliação para periódicos científicos on-line: proposta de indicadores bibliográficos e telemáticos. 2002. 210 f. Dissertação (Mestrado em Engenharia de Produção) - Programa de Pós-Graduação em Engenharia de Produção, Universidade Federal de Santa Catarina, Florianópolis, 2002.
[6] K.A.P.B. Duarte e R.S. Rodrigues, Periódicos em acesso aberto na área do direito. Revista Digital de Biblioteconomia e Ciência da Informação, Campinas, v. 9, n. 2 p.100-120, jan./jun. 2012.

[7] CAPES. Coordenação de Aperfeiçoamento de Pessoal de Nível Superior. Qualis Periódicos. Disponível em: <http// http://www.capes.gov.br/avaliacao/qualis > Vários acessos.

[8] C. Kieling e R.R.F. Gonçalves. Assessing the quality of a scientific journal: the case of Revista Brasileira de Psiquiatria. Revista Brasileira de Psiquiatria, v. 29, p. 177$181,2007$.

[9] B.K. Szymanski, J. L. De la Rosa and M. Krishnamoorthy, An Internet measure of the value of citations. Information Sciences, v. 185, n. 1, p. 18-31, 2012.

[10] P. Ingwersen. The calculation of web impact factors. Journal of Documentation, v. 54, n. 2, p. 236-243, 1998.

[11] L.A. Zadeh, Fuzzy Sets. Information and Control, v. 8, n. 3, p. 338-353, 1965.

[12] T.C. Wang and Y.H. Chen. Applying fuzzy linguistic preference relations to the improvement of consistency of fuzzy AHP. Information Sciences, v. 178, n. 19, p. 3755-3765, 2008

[13] T.L. Saaty and L.G. Vargas. The seven pillars of the Analytic Hierarchy Process: models, methods, concepts \& applications of the Analytic Hierarchy Process. In: (Ed.): Springer US, v.34, p.27-46, 2001

[14] W. Ho, P.K. Dey and M. Lockström. Strategic sourcing: a combined QFD and AHP approach in manufacturing. Supply Chain Management: An International Journal, v. 16 , n. 6 , p. 446-461, 2011.

[15] M. Bernasconi, C. Choirat and R. Seri. Empirical properties of group preference aggregation methods employed in AHP: theory and evidence. European Journal of Operational Research, v. 232, n. 3, p. 584-592, 2014.

[16] Y.-M. Wang, Y. Luo and Z. Hua. On the extent analysis method for fuzzy AHP and its applications. European Journal of Operational Research, v. 186, n. 2, p. 735$747,2008$.

[17] A.C. Kutlu and M. Ekmekçioğlu. Fuzzy failure modes and effects analysis by using fuzzy TOPSIS-based fuzzy AHP. Expert Systems with Applications, v. 39, n. 1, p. 61-67, 2012.

[18] D.-Y Chang. Applications of the extent analysis method on fuzzy AHP. European Journal of Operational Research, v. 95, n. 3, p. 649-655, 1996.

[19] C. Kahraman, U. Cebeci and D. Ruan. Multi-attribute comparison of catering service companies using fuzzy AHP: The case of Turkey. International Journal of Production Economics, v. 87, n. 2, p. 171-184, 2004.

[20] J. Cho and J. Lee. Development of a new technology product evaluation model for assessing commercialization opportunities using Delphi method and fuzzy AHP approach. Expert Systems with Applications, v. 40, n. 13, p. 5314-5330, 2013.

[21] Ying, Wang, Kyung-Ae, Jung, Gi-Tae, Yeo and Chien-Chang, Chou. Selecting a cruise port of call location using the fuzzy-AHP method: a case study in East Asia. Tourism Management, v. 42, p. 262-270, 2014.

[22] Y. Ju, A. Wang and X. Liu. Evaluating emergency response capacity by fuzzy AHP and 2-tuple fuzzy linguistic approach. Expert Systems with Applications, v. 39, n. 8, p. $6972-6981,2012$

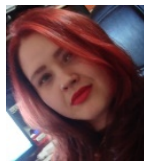

Nara Medianeira Stefano, Doutora em Engenharia de Produção (Universidade Federal de Santa Catariana - UFSC), Economista, Editora do Iberoamerican Journal of Industrial Engineering (IJIE). Atua em várias áreas tais como: gestão de custos, métodos quantitativos, capital intelectual, serviços, gestão do conhecimento. Maiores informações disponível em: http://lattes.cnpq.br/2043932840117404.

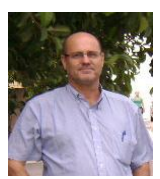

Nelson Casarotto Filho, Doutor em Engenharia de Produção, Professor na Universidade Federal de Santa Catarina (UFSC) no programa de pós-graduação em Engenharia de Produção. Maiores informações disponível em: http://lattes.cnpq.br/6910508438554107

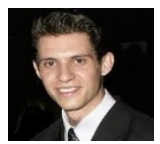

Rodrigo Barichello, Mestre em Engenharia de Produção, Doutorando em Engenharia de Produção na Universidade Federal de Santa Catarina (UFSC). Maiores informações disponível em: http://lattes.cnpq.br/6760700105264006 